\title{
Concurrent hyperfractionated chemoradiotherapy for head and neck squamous cell carcinoma: the prognostic impact of the overall treatment time and completion rates of chemotherapy
}

\author{
Masami Fujii ${ }^{1}$, Takayuki Ohguri ${ }^{\text {* }}$, Katsuya Yahara ${ }^{1}$, Hajime Imada ${ }^{2}$, Kyosuke Tomura ${ }^{1}$, Mai Sakagami ${ }^{1}$, \\ Gunji Nagatani ${ }^{3}$, Hideaki Suzuki ${ }^{3}$ and Yukunori Korogi ${ }^{1}$
}

\begin{abstract}
The purpose of this study was to investigate whether the overall treatment time and completion rates of chemotherapy were predictive factors for the survival rates in patients with squamous cell carcinoma of the head and neck (SCCHN) who were treated with concurrent chemoradiotherapy (CCRT) using hyperfractionated radiotherapy (RT) and daily carboplatin. The number of intermission days of RT were as follows; $0(n=37), 1-5(n=8), 6-10(n=12)$ and $\geq 11(n=12)$, and the days of RT without carboplatin; $0(n=27), 1-5(n=13), 6-10(n=13)$ and $\geq 7(n=16)$. The overall treatment time ( $\leq 48 \mathrm{vs} \geq 49$ days) was a significant prognostic factor for the local control, disease-free survival and overall survival rates. The completion rate of chemotherapy, as the number of days of RT without carboplatin, was not a significant factor affecting any of the survival rates. In discussion, the strengths of the present study contain that all the patients were treated with 72 Gy delivered as 1.2 Gy twice daily, and received concurrent chemotherapy comprising daily carboplatin as a radio-sensitizer. Based on the results, the completion rate of chemotherapy may have a lower impact on the local control rate in comparison with the overall treatment time. We believe that when a treatment interruption is needed because of the acute toxicities, hyperfractionated RT should be resumed as soon as possible independently while continuing the break of daily carboplatin. The overall treatment time influenced the clinical outcomes in SCCHN patients treated with hyperfractionated CCRT using carboplatin, while the impact of the completion rates of daily carboplatin was limited. Sixty-nine consecutive patients with SCCHN were initially treated with definitive CCRT and were retrospectively analyzed. All 69 patients were treated with CCRT using hyperfractionated RT of $72 \mathrm{~Gy}$ in 60 fractions and daily carboplatin $\left(25 \mathrm{mg} / \mathrm{m}^{2}\right)$. The patients treated with other chemotherapeutic regimens or induction chemotherapy were excluded. On the intermission days of the RT, carboplatin was not prescribed. After the intermission, CCRT using RT plus daily carboplatin or RT alone was resumed.
\end{abstract}

Keywords: Concurrent chemoradiotherapy, Head and neck cancer, Hyperfractionation, Carboplatin

\section{Background}

The prolongation of the overall treatment time of radiotherapy (RT) results in poorer clinical outcomes in

\footnotetext{
*Correspondence: ogurieye@med.uoeh-u.ac.jp

1 Department of Radiology, University of Occupational and Environmental

Health, 1-1 Iseigaoka, Yahatanishi-ku, Kitakyushu 807-8555, Japan

Full list of author information is available at the end of the article
}

patients with squamous cell carcinoma of the head and neck (SCCHN) (Duncan et al. 1996). Hyperfractionated RT has been used for head and neck cancers, especially for advanced cases, because it reduces the opportunity for the proliferation of tumor cells and increases the efficiency of tumor control without increasing late toxicity. Phase III randomized trials have demonstrated a 
significant improvement in the local control of head and neck cancers treated with twice-daily fractionation over once daily fractions (Horiot et al. 1992). Chemoradiotherapy has also been studied intensively in patients with SCCHN, (Marcial et al. 1990; Munro 1995). Concurrent chemoradiotherapy (CCRT) is attractive because chemotherapeutic agents may act as radiosensitizers, and the overall treatment time is not prolonged (Munro 1995). In the meta-analyses of head and neck cancers, the survival benefit of chemotherapy given synchronously with RT has been demonstrated (Munro 1995). In this context, concurrent chemoradiotherapy (CCRT) and hyperfractionated RT have been used for SCCHN, and the efficacy of CCRT using hyperfractionated RT was confirmed in a meta-analysis (Budach et al. 2006).

There have been only a few reports that have investigated whether the overall treatment time or completion rate of concurrent chemotherapy is a prognostic factor in SCCHN patients treated with CCRT using conventional fractionated RT (Pignon et al. 2000; Langendijk et al. 2004). However, to the best of our knowledge, there have been no reports that have evaluated the prognostic impact of the overall treatment time or completion rate of concurrent chemotherapy in patients treated with CCRT using hyperfractionated RT. The purpose of this study was therefore to investigate the prognostic impact of the overall treatment time and completion rates of chemotherapy in patients with SCCHN who were treated with CCRT using hyperfractionated RT.

\section{Results and discussion}

The follow-up ranged from 3 to 120 months (median, 46 months). Table 1 shows the number of intermission days of RT and days of RT without carboplatin. The 3 -year LC, DFS, DMFS and OS rates in all 69 patients were 66, 62, 90 and $75 \%$ respectively. Local recurrence and distant metastasis developed in 24 (39\%) and seven (10\%) patients, respectively.

Table 2 summarizes the results of the univariate analyses by the Kaplan-Meier approach with log-rank testing to evaluate the impact of certain factors on the survival rates. The number of overall treatment days was a significant prognostic factor for the LC, DFS and OS rates (Figs. 1, 2). The completion rate of chemotherapy, as the number of days of RT without carboplatin, was not a significant factor associated with any of the survival rates (Fig. 3). The clinical stage was a significant factor associated with the LC, DFS DMFS and OS. The T stage was also a significant predictor of the LC, DFS and OS.

In discussion, the present study is the first study to evaluate the overall treatment time of RT and the completion rates of chemotherapy for SCCHN patients treated with CCRT using hyperfractionated RT. The
Table 1 The intermission of RT and days of RT without carboplatin

\begin{tabular}{lcl}
\hline Days & $\begin{array}{l}\text { Intermission days } \\
\text { of RT } \mathbf{n}(\%)\end{array}$ & $\begin{array}{l}\text { Days of RT without } \\
\text { carboplatin } \mathbf{n}(\%)\end{array}$ \\
\hline 0 & $37(54)$ & $27(39)$ \\
$1-5$ & $8(12)$ & $13(19)$ \\
$6-10$ & $12(7)$ & $13(19)$ \\
$11-20$ & $11(16)$ & $13(19)$ \\
$21-$ & $1(1)$ & $3(4)$ \\
\hline
\end{tabular}

strengths of the present study include that all the patients were initially treated with 72 Gy delivered as 1.2 Gy twice daily, and received concurrent chemotherapy comprising daily carboplatin as a radio-sensitizer for the hyperfractionated RT (Chitapanarux et al. 2007). In addition, the patients who received neoadjuvant chemotherapy, followed by radiotherapy, were not included. Therefore, the effects of the overall treatment time and completion rates of daily carboplatin on the clinical outcomes could be evaluated with less bias. Our results confirmed that the overall treatment time was a significant factor predicting the LC, DFS and OS rates, while the completion rate of daily carboplatin was not.

In many of the previous studies of the clinical outcomes of RT alone, an interruption of RT was found to be a significant predictor of the local control (Bese et al. 2007). RT alone with a hyperfractionated schedule also showed a significant improvement in the LC and DFS in patients treated with shorter overall treatment times (Leborgne et al. 2001). However, as mentioned in the Introduction, there have been limited clinical reports about treatment interruption in SCCHN patients treated with CCRT (McCloskey et al. 2009; Gupta et al. 2009). McCloskey et al. reported that RT treatment interruption greater than 1 week was a significant predictor of worse LC after definitive CCRT using cisplatin (most common regimen $100 \mathrm{mg} / \mathrm{m}^{2}$ on days 1,22 and 43 ) and conventional fractionated RT (McCloskey et al. 2009). Gupta et al. demonstrated that the total cycles ( $\geq 6$ cycles) of concurrent weekly cisplatin $\left(30 \mathrm{mg} / \mathrm{m}^{2}\right)$, as well as a shorter overall treatment time, was a significant prognostic factors for a better LC and DFS (Gupta et al. 2009). In the current study of CCRT using hyperfractionated RT and daily carboplatin, although the rate of completion of chemotherapy was not a prognostic factor, the overall treatment time was a significant predictor. We supposed that the completion rate of chemotherapy may have a lower impact on the LC rate in comparison with the overall treatment time, because the daily low-dose regimen was prescribed mainly as a radio-sensitizer, and the antitumor effects of hyperfractionated RT may strongly depend on the overall treatment time (Leborgne et al. 2001). 
Table 2 The results of the univariate analyses of factors predicting the survival rates

\begin{tabular}{|c|c|c|c|c|c|c|c|c|c|}
\hline & \multirow[t]{2}{*}{$\mathrm{n}$} & \multicolumn{2}{|c|}{ Local control } & \multicolumn{2}{|c|}{ Disease-free survival } & \multicolumn{2}{|c|}{$\begin{array}{l}\text { Distant metastasis-free } \\
\text { survival }\end{array}$} & \multicolumn{2}{|c|}{ Overall survival } \\
\hline & & 3-year (\%) & $p$ & 3-year (\%) & $p$ & 3-year (\%) & $p$ & 3-year (\%) & $p$ \\
\hline \multicolumn{2}{|l|}{ Age (years) } & & 0.41 & & 0.44 & & 0.81 & & 0.82 \\
\hline$<65$ & 33 & 72 & & 67 & & 87 & & 79 & \\
\hline$\geq 65$ & 36 & 62 & & 59 & & 91 & & 70 & \\
\hline \multicolumn{2}{|l|}{ PS } & & 0.73 & & 0.63 & & 0.83 & & 0.34 \\
\hline $0-1$ & 59 & 68 & & 64 & & 87 & & 77 & \\
\hline $2-3$ & 10 & 64 & & 58 & & 100 & & 61 & \\
\hline \multicolumn{2}{|l|}{ Clinical stage } & & 0.007 & & 0.0009 & & 0.01 & & $<0.0001$ \\
\hline$|-| \mid$ & 35 & 84 & & 84 & & & & 100 & \\
\hline III-IV & 34 & 50 & & 39 & & & & 49 & \\
\hline \multicolumn{2}{|l|}{ Tumor stage } & & $<0.0001$ & & $<0.0001$ & & 0.17 & & $<0.0001$ \\
\hline $\mathrm{T} 1-2$ & 55 & 79 & & 74 & & 80 & & 87 & \\
\hline T3-4 & 14 & 23 & & 17 & & 90 & & 34 & \\
\hline \multicolumn{2}{|l|}{ N stage } & & 0.05 & & 0.0091 & & 0.01 & & 0.0012 \\
\hline No & 38 & 77 & & 77 & & 97 & & 94 & \\
\hline N1-3 & 31 & 54 & & 43 & & 76 & & 52 & \\
\hline \multicolumn{3}{|c|}{ Overall treatment (days) } & 0.039 & & 0.015 & & 0.11 & & 0.02 \\
\hline$\leq 48$ & 45 & 78 & & 74 & & 91 & & 84 & \\
\hline$\geq 49$ & 24 & 48 & & 41 & & 85 & & 56 & \\
\hline \multicolumn{3}{|c|}{ Days of RT without carboplatin } & 0.69 & & 0.61 & & 0.82 & & 0.21 \\
\hline$\leq 5$ & 40 & 68 & & 64 & & 83 & & 80 & \\
\hline$\geq 6$ & 29 & 64 & & 41 & & 95 & & 67 & \\
\hline \multicolumn{3}{|c|}{ Hemoglobin at the start of RT ( $\mathrm{g} / \mathrm{dl}$ ) } & 0.30 & & 0.22 & & 0.66 & & 0.02 \\
\hline$<12$ & 18 & 51 & & 44 & & 83 & & 57 & \\
\hline$\geq 12$ & 51 & 71 & & 68 & & 92 & & 82 & \\
\hline
\end{tabular}

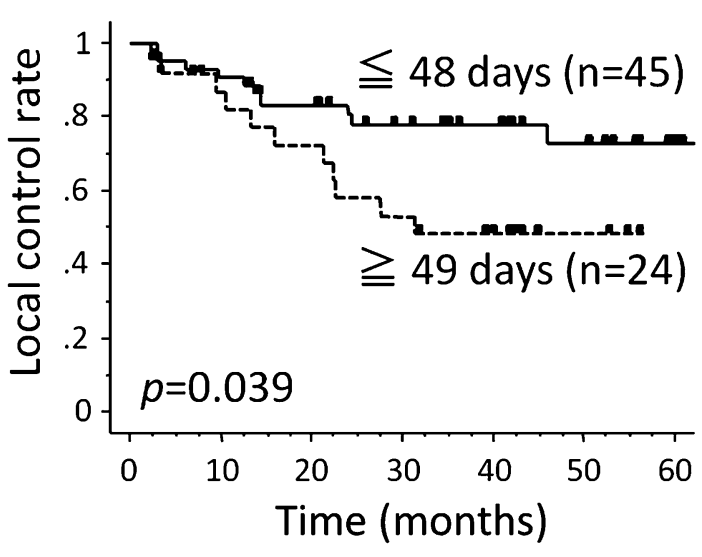

Fig. 1 The overall treatment period ( $\leq 48$ days) was a significant predictor of the local control rate $(p=0.039)$

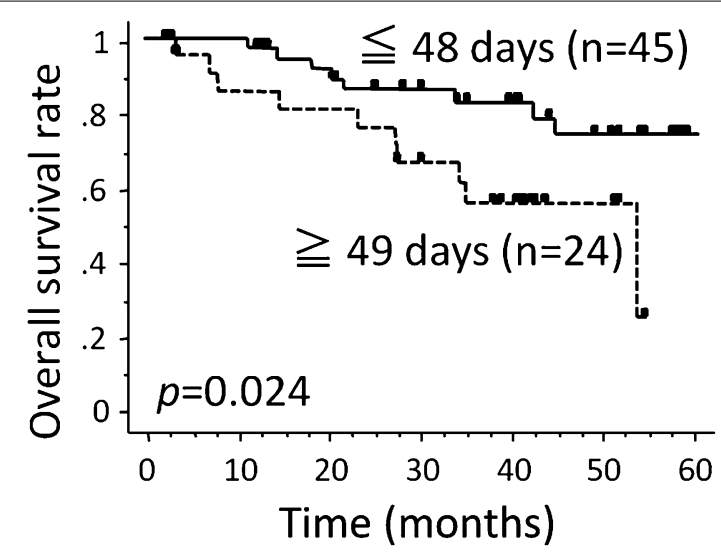

Fig. 2 The overall treatment period ( $\leq 48$ days) was a significant predictor of the overall survival rate $(p=0.024)$
A prolonged overall treatment time is a well-known factor that has a negative impact on the outcome of SCCHN due to tumor cell repopulation (Hoffstetter et al. 1997; Tarnawski et al. 2002; Suwinski et al. 2003).
Nishimura et al. reported that an overall treatment time longer than 49 days was significantly associated with a poor local control rate in patients with T1 or T2 glottic carcinoma; a 1-week interruption of RT significantly 


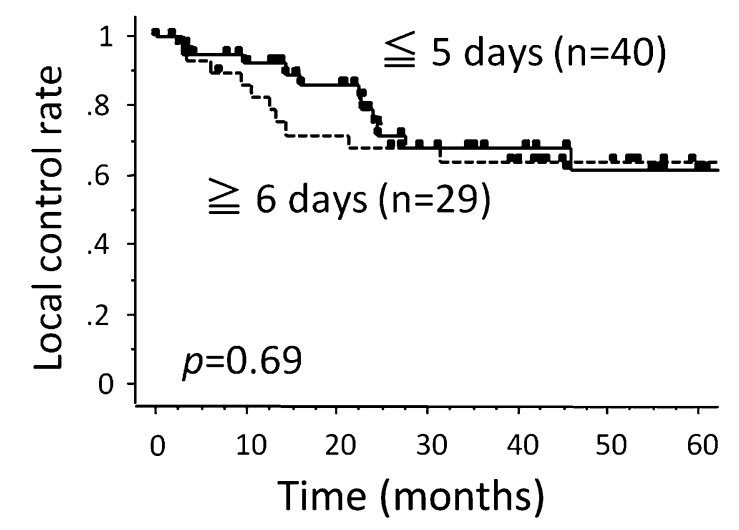

Fig. 3 The rate of completion of chemotherapy, as the number of days of RT without carboplatin, was not a significant predictor of the local control rate

reduced the 5-year local control probability of T1 glottic tumors from 89 to $74 \%$ (Nishimura et al. 1996). Kawashima et al. also indicated that the patients who had received $70 \mathrm{~Gy} / \leq 49$ days for accelerated fractionation RT achieved a better local control rate than those who had received other, more conservative total dose/overall treatment time with statistical significance in patients with T2 or worse hypopharyngeal cancer (Kawashima et al. 2007). According to these clinical results, we chose $\leq 49$ and $\geq 49$ days as the difference in treatment time, and recommended that patients receive radiation therapy for a maximum of 48 days and no longer, because we confirmed that the overall treatment time ( $\leq 49$ days) was a significant prognostic factor for the survival rates in the current study.

Hyperfractionated RT has resulted in increased severe acute complications requiring intensive nutritional support for the completion of treatment in some patients. Wang et al. suggested that a prolongation of the treatment gap for more than 14 days adversely affected the local control in patients with locally advanced head and neck cancer treated with accelerated hyperfractionated radiation therapy, and the midcourse treatment gap should be kept as short as possible (Wang et al. 1996). In a Phase III trial of hyperfractionated RT with or without concurrent chemotherapy for locally advanced head and neck cancer, combined treatment for advanced head and neck cancer was more efficacious than hyperfractionated RT alone (Brizel et al. 1998). This trial included planned treatment breaks to avoid the withdrawal of the recruited subjects due to acute toxicity; eventually, the treatment time of RT in the hyperfractionated RT arm averaged 42 days, while that in the hyperfractionated RT plus concurrent chemotherapy arm averaged 47 days (Brizel et al. 1998). However, the long-term follow-up in the Phase III Radiation Therapy Oncology Group (RTOG)
0129 trial indicated no difference in the overall survival or late toxicity with the use of accelerated-hyperfractionated vs standard RT plus cisplatin in patients with locally advanced head and neck cancer (Nguyen-Tan et al. 2014). Based on the results of the present study of CCRT using hyperfractionated RT and daily carboplatin, we believe that when a treatment interruption is needed because of the acute toxicities, hyperfractionated RT should be resumed as soon as possible independently while continuing the break of daily carboplatin.

Regarding the limitations associated with this study, it was a retrospective case series, so the possibility that there was some selection bias with regard to the prognostic factors could not be ruled out, although all of the patients received definitive CCRT using hyperfractionated RT of 70 Gy in 60 fractions and daily carboplatin, and were initially treated with non-surgical management. The treatment regimen of CCRT using hyperfractionated RT and daily carboplatin differed from the standard therapy, such as CCRT using conventional fractionation and cisplatin. However, a Phase III randomized trial of CRT using cisplatin vs. carboplatin for head and neck cancer demonstrated that there was no difference in the survival rates between cisplatin and carboplatin, and the completion rates of carboplatin was higher than that of cisplatin (Chitapanarux et al. 2007). In addition, a significant improvement in the local control of head and neck cancers treated with twice-daily fractionation over once daily fractions in Phase III randomized trials for RT monotherapy for head and neck cancer was observed (Horiot et al. 1992). Therefore, we chose this regimen presuming an improvement in the treatment results in patients with $\mathrm{SCCHN}$. A formal prospective trial is necessary to determine the prognostic impact of the overall treatment time and completion rate of chemotherapy in patients treated with CCRT using hyperfractionated RT.

\section{Conclusions}

In conclusion, this study demonstrated that the longer overall treatment time may have a negative impact on the clinical outcomes in SCCHN patients treated with concurrent CCRT using hyperfractionated RT and daily carboplatin, while the completion rates of daily carboplatin may not affect the clinical outcomes. When the acute toxicities that need the treatment interruption occur, it is important to consider the early resume of hyperfractionated RT without restart of the daily carboplatin.

\section{Methods}

From June 1999 to January 2010, 608 consecutive patients with primary head and neck cancer were prospectively recorded in the database for RT at the authors' institution. The following requirements had to be met for 
patients to be included in this retrospective study: a pathologically confirmed SCCHN, treated with concurrent chemoradiotherapy (CCRT) using hyperfractionated RT of $72 \mathrm{~Gy}$ in 60 fractions and daily carboplatin $\left(25 \mathrm{mg} / \mathrm{m}^{2}\right)$, without distant metastatic disease. The following patients were excluded: those who had been treated with neoadjuvant chemotherapy, had received transoral debulking microsurgery and those who had postoperative disease. Sixty-nine patients were selected from the database and were retrospectively analyzed. Written informed consent for treatment was obtained from all patients. The study was approved by the Institutional Review Board of the authors' institution.

The characteristics of the patients are listed in Table 3. The performance status and tumor invasion were evaluated at the start of our treatment. Staging was achieved by clinical examination, $\mathrm{CT}$ and endoscopic examination, and in some cases, magnetic resonance imaging (MRI) and 18-Fluoro-deoxyglucose positron emission tomography (FDG-PET). All 69 patients were treated with two daily fractions of radiation of $1.2 \mathrm{~Gy}$ (total dose, 72 Gy), with a 6-h interval between fractions. Computed tomography-assisted three-dimensional treatment planning (Xio or FOCUS; CMS Japan, Tokyo, Japan) was used to determine the radiation fields. Uninvolved nodal areas of the neck were treated with $43.2 \mathrm{~Gy} / 36 \mathrm{Fr}$. The primary tumor and gross nodal disease were treated with 72 Gy/60 Fr. Prophylactic neck irradiation for uninvolved nodal areas was not performed in 14 patients with T2NOM0 glottic cancer of the larynx.

The detailed irradiation and concurrent chemotherapy schedules are shown in Fig. 4. Carboplatin was planned to be administered as an intravenous bolus immediately before the first daily fraction at a dose of $25 \mathrm{mg} / \mathrm{m}^{2}$ on every treatment day. On the intermission days of the hyperfractionated RT, carboplatin was not prescribed. After the intermission, CCRT using hyperfractionated RT plus daily carboplatin or hyperfractionated RT alone was resumed.

In all patients, complete head and neck examinations, including flexible laryngoscopy, were performed after the completion of CCRT and every 1-3 months for the first year, every $2-3$ months for the second and third years and every 2-6 months for the fourth and fifth years after the CCRT. CT scans were performed every 3-6 months at the discretion of the clinician, and in some patients, fluorodeoxyglucose positron emission tomography/computed tomography (FDG-PET/CT) or magnetic resonance imaging (MRI) was additionally used.

The local control (LC), defined as failure to have a recurrence within the radiation field, the disease-free survival (DFS), distant metastasis-free survival (DMFS) and overall survival (OS) rates were calculated from the

\section{Table 3 The patient characteristics}

\begin{tabular}{|c|c|}
\hline Variable & $n=69(\%)$ \\
\hline \multicolumn{2}{|l|}{ Age (years) } \\
\hline Median (range) & $69(40-85)$ \\
\hline \multicolumn{2}{|l|}{ Gender } \\
\hline Male & $60(87)$ \\
\hline Female & $9(13)$ \\
\hline \multicolumn{2}{|l|}{ Performance status } \\
\hline 0 & $12(17)$ \\
\hline 1 & $46(67)$ \\
\hline 2 & $10(15)$ \\
\hline 3 & $1(1)$ \\
\hline \multicolumn{2}{|l|}{ Tumor type, TNM ${ }^{a}$} \\
\hline Nasopharyngeal ca. & $5(7)$ \\
\hline T1N2M0 & 1 \\
\hline T1N3M0 & 1 \\
\hline $\mathrm{T} 2 \mathrm{~N} 1 \mathrm{M} 0$ & 3 \\
\hline Oropharyngeal ca. & $15(22)$ \\
\hline T1N0M0 & 1 \\
\hline T1N2M0 & 4 \\
\hline T2NOMO & 1 \\
\hline T2N1M0 & 2 \\
\hline $\mathrm{T} 2 \mathrm{~N} 2 \mathrm{MO}$ & 1 \\
\hline T3N1M0 & 1 \\
\hline T4N1M0 & 1 \\
\hline T4N2M0 & 1 \\
\hline T4N3M0 & 3 \\
\hline Hypopharyngeal ca. & $22(32)$ \\
\hline T1N0M0 & 2 \\
\hline $\mathrm{T} 1 \mathrm{~N} 2 \mathrm{M} 0$ & 1 \\
\hline T2NOMO & 8 \\
\hline T2N1M0 & 1 \\
\hline $\mathrm{T} 2 \mathrm{~N} 2 \mathrm{MO}$ & 4 \\
\hline T3N2M0 & 2 \\
\hline T4NOMO & 2 \\
\hline T4N2M0 & 2 \\
\hline Laryngeal ca. & $27(39)$ \\
\hline T2NOMO & 22 \\
\hline T2N1M0 & 1 \\
\hline $\mathrm{T} 2 \mathrm{~N} 2 \mathrm{MO}$ & 2 \\
\hline T3N0MO & 1 \\
\hline T3N2M0 & 1 \\
\hline
\end{tabular}

a International Union Against Cancer tumor, node, metastasis classification, 6th edition

start of RT using the Kaplan-Meier method. The statistical significance of the differences between the actuarial curves was assessed using the log-rank test. To identify prognostic factors for the survival rates, univariate analyses were performed using the age, performance status, clinical stage, $\mathrm{T}$ stage, $\mathrm{N}$ stage, overall treatment days, 
Timing of hyperfractionated RT and CBDCA

\section{CBDCA (daily $25 \mathrm{mg} / \mathrm{m}^{2}, 5$ times per week)}

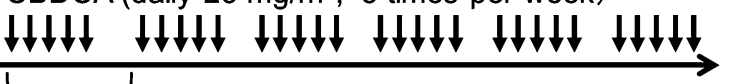
one week

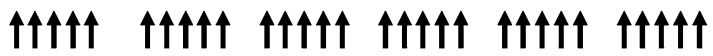

Hyperfractionated RT (total dose 72Gy)

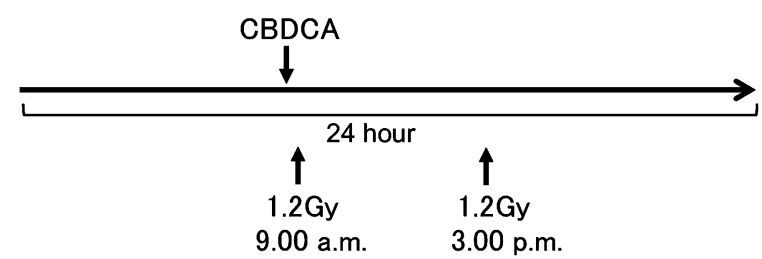

Fig. 4 The schedules of concurrent chemoradiotherapy using hyperfractionated radiotherapy and carboplatin

days of RT without carboplatin and the hemoglobin level at the start of RT. Multivariate analysis was not performed because of the small number of patients.

\section{Authors' contributions}

MF collected and analyzed data, and drafted the manuscript; TO analyzed data and drafted the manuscript; KY collected and analyzed data; HI, KT, MS, GN contributed to data analysis; HS, YK revised the manuscript. All authors read and approved the final manuscript.

\section{Author details}

${ }^{1}$ Department of Radiology, University of Occupational and Environmental Health, 1-1 Iseigaoka, Yahatanishi-ku, Kitakyushu 807-8555, Japan. ${ }^{2}$ Department of Cancer Therapy Center, Tobata Kyoritsu Hospital, Kitakyushu, Japan. ${ }^{3}$ Department of Otorhinolaryngology-Head and Neck Surgery, University of Occupational and Environmental Health, Kitakyushu, Japan.

\section{Compliance with ethical guidelines}

\section{Competing interests}

The authors declare that they have no competing interests.

\section{Informed consent}

Informed consent was obtained from all individual participants included in the study.

Received: 7 March 2015 Accepted: 14 August 2015

Published online: 25 August 2015

\section{References}

Bese NS, Munzuroglu F, Uslu B, Arbak S, Yesiladali G, Sut N et al (2007) Vitamin E protects against the development of radiation-induced pulmonary fibrosis in rats. Clin Oncol (R Coll Radiol) 19:260-264

Brizel DM, Albers ME, Fisher SR, Scher RL, Richtsmeier WJ, Hars V et al (1998) Hyperfractionated irradiation with or without concurrent chemotherapy for locally advanced head and neck cancer. N Engl J Med 338:1798-1804

Budach W, Hehr T, Budach V, Belka C, Dietz K (2006) A meta-analysis of hyperfractionated and accelerated radiotherapy and combined chemotherapy and radiotherapy regimens in unresected locally advanced squamous cell carcinoma of the head and neck. BMC Cancer 6:28
Chitapanarux I, Lorvidhaya V, Kamnerdsupaphon P, Sumitsawan Y, Tharavichitkul E, Sukthomya V et al (2007) Chemoradiation comparing cisplatin versus carboplatin in locally advanced nasopharyngeal cancer: randomised, non-inferiority, open trial. Eur J Cancer 43:1399-1406

Duncan W, MacDougall RH, Kerr GR, Downing D (1996) Adverse effect of treatment gaps in the outcome of radiotherapy for laryngeal cancer. Radiother Oncol 41:203-207

Gupta T, Agarwal JP, Ghosh-Laskar S, Parikh PM, D'Cruz AK, Dinshaw KA (2009) Radical radiotherapy with concurrent weekly cisplatin in loco-regionally advanced squamous cell carcinoma of the head and neck: a singleinstitution experience. Head Neck Oncol 1:17

Hoffstetter S, Marchal C, Peiffert D, Luporsi E, Lapeyre M, Pernot M et al (1997) Treatment duration as a prognostic factor for local control and survival in epidermoid carcinomas of the tonsillar region treated by combined external beam irradiation and brachytherapy. Radiother Oncol 45:141-148

Horiot JC, Le Fur R, N'Guyen T, Chenal C, Schraub S, Alfonsi S et al (1992) Hyperfractionation versus conventional fractionation in oropharyngeal carcinoma: final analysis of a randomized trial of the EORTC cooperative group of radiotherapy. Radiother Oncol 25:231-241

Kawashima M, Hayashi R, Tahara M, Yamazaki M, Miyazaki M, Arahira S et al (2007) Accelerated radiotherapy and larynx preservation in favorablerisk patients with T2 or worse hypopharyngeal cancer. Jpn J Clin Oncol 37:345-352

Langendijk JA, Leemans CR, Buter J, Berkhof J, Slotman BJ (2004) The additional value of chemotherapy to radiotherapy in locally advanced nasopharyngeal carcinoma: a meta-analysis of the published literature. J Clin Oncol 22:4604-4612

Leborgne F, Leborgne JH, Fowler J, Zubizarreta E, Mezzera J (2001) Accelerated hyperfractionated irradiation for advanced head and neck cancer: effect of shortening the median treatment duration by 13 days. Head Neck 23:661-668

Marcial VA, Pajak TF, Mohiuddin M, Cooper JS, al Sarraf M, Mowry PA et al (1990) Concomitant cisplatin chemotherapy and radiotherapy in advanced mucosal squamous cell carcinoma of the head and neck. Long-term results of the Radiation Therapy Oncology Group study 81-17. Cancer 66:1861-1868

McCloskey SA, Jaggernauth W, Rigual NR, Hicks WL Jr, Popat SR, Sullivan M et al (2009) Radiation treatment interruptions greater than one week and low hemoglobin levels $(12 \mathrm{~g} / \mathrm{dL}$ ) are predictors of local regional failure after definitive concurrent chemotherapy and intensity-modulated radiation therapy for squamous cell carcinoma of the head and neck. Am J Clin Oncol 32:587-591

Munro AJ (1995) An overview of randomised controlled trials of adjuvant chemotherapy in head and neck cancer. Br J Cancer 71:83-91

Nguyen-Tan PF, Zhang Q, Ang KK, Weber RS, Rosenthal DI, Soulieres D et al (2014) Randomized phase III trial to test accelerated versus standard fractionation in combination with concurrent cisplatin for head and neck carcinomas in the Radiation Therapy Oncology Group 0129 trial: longterm report of efficacy and toxicity. J Clin Oncol 32:3858-3866

Nishimura Y, Nagata Y, Okajima K, Mitsumori M, Hiraoka M, Masunaga S et a (1996) Radiation therapy for T1, 2 glottic carcinoma: impact of overall treatment time on local control. Radiother Oncol 40:225-232

Pignon JP, Bourhis J, Domenge C, Designe L (2000) Chemotherapy added to locoregional treatment for head and neck squamous-cell carcinoma: three meta-analyses of updated individual data. MACH-NC Collaborative Group. Meta-Analysis of Chemotherapy on Head and Neck Cancer. Lancet 355:949-955

Suwinski R, Sowa A, Rutkowski T, Wydmanski J, Tarnawski R, Maciejewski B (2003) Time factor in postoperative radiotherapy: a multivariate locoregional control analysis in 868 patients. Int J Radiat Oncol Biol Phys 56:399-412

Tarnawski R, Fowler J, Skladowski K, Swierniak A, Suwinski R, Maciejewski B et al (2002) How fast is repopulation of tumor cells during the treatment gap? Int J Radiat Oncol Biol Phys 54:229-236

Wang CC, Efird J, Nakfoor B, Martins P (1996) Local control of T3 carcinomas after accelerated fractionation: a look at the "gap". Int J Radiat Oncol Biol Phys 35:439-441 